\title{
Factores que inciden en la selección de marca de cerveza en la ciudad de cali
}

\section{Factors influencing beer brand selection in the city of cali}

DOI: $10.46932 / \mathrm{sfjdv2n5-096}$

Received in: Oct 1st, 2021

Accepted in: Dec 30th, 2021

Julián Andrés Manrique Aristizabal

Msc. Ciencia Cognitiva

Universidad Autónoma de Occidente

Calle 25 \# 115 - 85 Vía Cali - Jamundí

E-mail: jamanrique@uao.edu.co

Valentina Bernat

Bachelor. Mercadeo y negocios internacionales

Universidad Autónoma de Occidente

Calle 25 \# 115 - 85 Vía Cali - Jamundí

E-mail: valentina.bernat@uao.edu.co

Victoria Montaño

Bachelor. Mercadeo y negocios internacionales

Universidad Autónoma de Occidente

Calle 25 \# 115 - 85 Vía Cali - Jamundí

E-mail: maria.montano@uao.edu.co

\begin{abstract}
RESUMEN
La evolución del consumo de cerveza en Colombia ha tomado relevancia en las últimas décadas. Para indagar acerca de los factores que inciden en la selección de una marca de cerveza en la ciudad de Cali, se realizó una investigación de mercados descriptiva con un diseño mixto. Los participantes de dos grupos focales no lograron reconocer el sabor de la mayoría de marcas de cerveza que degustaron en una cata a ciegas. Al indagar por medio de encuestas a 484 sujetos de 18 a 59 años de edad pertenecientes a diferentes estratos socioeconómicos, se aprecia cierta correspondencia entre las marcas favoritas y el segmento al que va dirigida. Los resultados sugieren que, en la selección de cervezas, la percepción del sabor como atributo del producto no es tan importante como la adherencia a una marca favorita, lo cual podría explicarse por el trabajo de branding que realiza cada marca.
\end{abstract}

Palabras clave: Comportamiento del consumidor, top of mind, lealtad de marca, valor de marca.

\begin{abstract}
The evolution of beer consumption in Colombia has become relevant in recent decades. To inquire about the factors that influence the selection of a beer brand in the city of Cali, a descriptive market research was carried out with a mixed design. Participants in two focus groups failed to recognize the taste of most beer brands they tasted in a blind tasting test. When inquiring through surveys to 485 subjects between 18 and 59 years of age belonging to different socioeconomic strata, a certain correspondence between the favorite brands and the segment to which it is addressed is appreciated. The results suggest that, in the selection of beers, the perception of flavor as an attribute of the product is not as strong as the adherence to a favorite brand, which could be explained by the branding work carried out by each brand.
\end{abstract}


Keywords: Consumer behavior, top of mind, brand loyalty, brand equity.

\section{INTRODUCCIÓN}

La evolución del consumo de cerveza ha tomado relevancia en las últimas décadas. En Colombia, el consumo per cápita promedio de cerveza en el 2019 fue de 54,8 litros anuales, lo que correspondió al $73 \%$ del gasto en la categoría de licores; un consumo que está un 35\% por encima del de rones, aguardientes o vinos (La República, 2019; Euromonitor, 2019). Si bien se espera que las ventas de cerveza on-trade bajen en un 32\% debido al impacto por el COVID-19, las ventas para consumir en otro lugar distinto al que se compra (off-trade) podrían aumentar un $2 \%$, concentrando las ganancias de este negocio en los supermercados e hipermercados distribuidores (Euromonitor, 2020). De esta forma, según el informe de Euromonitor, uno de los mayores cambios que ha traído la pandemia del coronavirus a la categoría de cervezas ha sido un mayor impacto en las cervezas artesanales y tipo Premium pues las grandes cervecerías, con sus marcas populares, estarían en mejores condiciones para distribuir el producto a precios bajos.

Teniendo en cuenta que la fidelización es imprescindible para la rentabilidad y el posicionamiento de una marca, especialmente en un mercado saturado donde la competencia es relevante, resulta importante indagar acerca de los factores que inciden en la selección de una marca de cerveza. En este estudio, se realizó una investigación de mercados descriptiva con diseño mixto con el fin de retratar la lealtad de marca del consumo de cerveza de jóvenes y adultos de 18 a 59 años de edad de estratos 1,2,3,4,5 y 6 de la ciudad de Cali.

\section{REVISIÓN DE LA LITERATURA}

\subsection{COMPORTAMIENTO DEL CONSUMIDOR}

Salomon (2008) define el comportamiento del consumidor como "el estudio de los procesos que intervienen cuando una persona o grupo selecciona, compra, usa o desecha productos, servicios, ideas o experiencias para satisfacer necesidades y deseos" (p. 7). Esta definición va enfocada hacia la comprensión de la toma de decisiones de las personas en los diferentes entornos donde las necesidades y deseos que se quieren satisfacer ayudan a definir sus gustos y preferencias.

Cabe resaltar que cada consumidor es único y diferente, pues su comportamiento hacia la toma de decisiones puede variar según factores externos e internos que influyen en el proceso de compra. Autores como Schiffman y Lazar (2010) y Kotler y Armstrong (2013), exponen un modelo de toma de decisiones del consumidor dividido en tres fases fundamentales; la fase de entrada consiste en el reconocimiento de la necesidad de un producto a través de las campañas de marketing e influencias socioculturales externas; 
en la fase del proceso de compra entran a operar los factores psicológicos de cada individuo (percepción, motivación, personalidad, actitudes, aprendizaje), los cuales le permiten interpretar esos estímulos externos e inciden en que indague más acerca del producto y evalúe las posibles alternativas que puede tomar. Al tomar la decisión de realizar la compra, el consumidor entra a la fase de salida que determina si la compra fue satisfactoria en función de comparar el beneficio esperado y el obtenido al consumar el producto.

\subsection{LEALTAD DE MARCA}

Las marcas que realizan esfuerzos de marketing están comprometidas con el consumidor a mantener la identidad de la marca justo como ellos la conocieron. Es claro que todas las marcas deben evolucionar e innovar en todos los aspectos, pero su esencia debe permanecer. La marca debe conocer al cliente y saber qué es lo que más aprecia de ella, así como también su comportamiento frente a la misma. Aaker (2007; 2015), propone que si la percepción de los consumidores de la marca es buena, la empresa obtendrá lealtad por su parte y por consiguiente, las utilidades aumentarán ya que los consumidores se vuelven más insensibles a cambios en la política de precios de una marca cuando consideran que ésta tiene un alto valor.

Keller (1993), define el valor de marca como el efecto añadido que el conocimiento de marca ejerce sobre el consumidor como resultado de las inversiones en marketing. De acuerdo con el modelo de customer-based brand equity (CBBE, por sus siglas en inglés) propuesto por Aaker (1996), Keller (1993), Tolba y Hassan (2006), citado en González, Orozco y Barrios (2011), existen una serie de factores cognitivos y afectivos afines al valor de marca que intervienen en el proceso de compra. Para los autores, el conocimiento de marca se mide y calcula según la notoriedad, la cual afecta la capacidad de elección pues los consumidores tienen en su mente una serie de marcas de distintas categorías de productos y preferirá aquella con mayor reconocimiento en el mercado. Esto contribuye a una enorme ventaja competitiva para las marcas que están bien posicionadas, asegurando la lealtad de marca por parte de sus clientes. Para Roberts (2004; 2006), las marcas se valoran no solo por su notoriedad, la esencia del top of mind (ToM), sino por el amor que puedan inspirar, por lo que su poder radica en cultivar una relación personal única con el consumidor, lo que se conoce como el top of Heart (ToH).

\section{METODOLOGÍA}

El presente estudio se concibe como una investigación concluyente descriptiva con un diseño mixto transversal, cuyo trabajo de campo se desarrolló a finales del 2019 en dos fases: En la primera fase (cualitativa), se realizaron dos grupos focales conformados por 10 personas, hombres y mujeres de 18 a 30 años. Además de inquirir sobre la disposición actitudinal y comportamental de los participantes 
respecto el consumo de cerveza, se realizó una cata a ciegas administrándoles un vaso oscuro de plástico de 2 onzas, lleno a la mitad. Los vasos fueron marcados en su exterior con un número del 1 al 6 , correspondientes a las siguientes marcas de cerveza: Póker, Águila, Águila Light, Club Colombia. Andina y Redd's. Las cervezas (en lata) se encontraban en un contenedor con hielo antes de ser depositadas en los vasos y aunque la correspondencia marca-número era conocida para los investigadores, los vasos fueron administrados aleatoriamente a los participantes, quienes debían escribir individualmente en una hoja que contenía los logos de las marcas, el número en el vaso (1-6) que creían correspondía con la marca, inmediatamente después de probarla. En la segunda fase (cuantitativa), mediante un muestreo estratificado se realizaron 485 encuestas a personas de 18 a 59 años de edad teniendo en cuenta un único criterio de inclusión: que hubiesen consumido y/o comprado cerveza. Algunas encuestas fueron diligenciadas presencialmente en barrios de diferentes estratos de la ciudad de Cali y otras distribuidas por medio de formularios de google.

\section{RESULTADOS}

En la segunda fase participaron 485 personas de la ciudad de Cali-Colombia, 52,8\% mujeres y 47,2\% hombres, con una edad media de 30.4 años y desviación estándar de 11.0 (Rango: 18-59 años). La distribución por nivel socioeconómico (NSE) fue la siguiente: NSE1 (16.5\%), NSE2 (16.5\%), NSE3 (20.8\%), NSE4 (24.1\%), NSE5 (14.2\%) y NSE6 (7.8\%).

\subsection{TOP OF MIND Y TOP OF HEART}

En la figura 1 se aprecian los porcentajes de recordación tanto del ToM como del ToH. El top of mind fue evaluado a través de la pregunta: "si usted piensa en cerveza ¿qué marca se le viene a la mente?" Póker (40.2\%), Águila (19.6\%), Corona (18.1\%), Club Colombia (7.8\%) y Andina (4.7\%), fueron las marcas más recordadas por la población caleña. Así, aunque esta última obtuvo en el 2020 un reconocimiento como producto innovador del año en la categoría de cervezas (La Republica, 2020), la mayoría de los encuestados de la muestra posicionan a Andina en los últimos lugares, tal vez debido a su ingreso reciente al mercado. Se observa tambien en la muestra, la preferencia por una cerveza Premium importada (Corona) que por una Premium nacional (Club Colombia.) Al analizar el top of heart (" ¿cuál es su cerveza favorita?”) en función del género, se hallaron diferencias significativas $\left(\chi^{2}=78,861\right.$; p=0.000), observando que los hombres pensaron en Póker, Águila, Corona y Club Colombia, mientras las mujeres en Águila, Póker, Corona y Redd's, respectivamente. Respecto la fuerza de asociación entre las variables (género x marca de cerveza), se obtuvo un coeficiente V de Cramer $=0.403$ ( $\mathrm{p}<0.000$ ), lo que indica que la asociación entre las variables es moderada. No se encontraron diferencias significativas al cruzar el estrato con el top of mind $\left(\chi^{2}=98,173 ; p=0.261\right)$, pero si con el top of heart $\left(\chi^{2}=276,112\right.$; 
$\mathrm{p}=0.000$ ). Águila es la favorita en el estrato 1, Póker en el estrato 2 y 4, Corona en el 3, mientras que los estratos 5 y 6 prefieren otro tipo de cervezas (importadas, artesanales y otras marcas Premium). Respecto la edad, la cual se agrupó con base en la media de la muestra en "jóvenes" (<30 años, 58\% de los participantes) y "adultos" (>30, 42\% de los participantes), no se observan diferencias significativas en el top of mind $\left(\chi^{2}=33,66 ; p=0.14\right)$ pero si en el top of heart $\left(\chi^{2}=79,89 ; p=0.000\right)$, encontrándose que aunque ambos grupos recuerdan Póker y Águila en mayor medida, los adultos prefieren más la Póker y los jóvenes la Corona.

Tabla 1. Top of Mind (ToM) y Top of Heart (ToH) de marcas de cerveza en Cali

\begin{tabular}{|c|c|c|}
\hline Marca & ToM $(\boldsymbol{\%})$ & ToH (\%) \\
\hline Póker & 40,2 & 26,8 \\
\hline Águila & 19,6 & 19,3 \\
\hline Corona & 18,1 & 15,7 \\
\hline Club Colombia & 7,8 & 10,3 \\
\hline Andina & 4,7 & 0.8 \\
\hline Águila Light & 0.6 & 8,1 \\
\hline Otras & 9,6 & 19 \\
\hline
\end{tabular}

\subsection{FACTOR DE DECISIÓN}

El factor de decisión reúne los atributos que los consumidores esperan que tenga el producto para su posterior adquisición. Como se puede apreciar en la figura 1, en la encuesta se incluyeron como variables: marca, precio, sabor, temperatura y porcentaje de alcohol. En este caso, no se hallaron diferencias significativas respecto el género $\left(\chi^{2}=7,795 ; p=0.99\right)$ o la edad $\left(\chi^{2}=7,47 ; p=0.11\right)$ pero si con el estrato $\left(\chi^{2}=45,299 ; p=0.001\right)$. En general, los participantes refieren que la marca no representa un factor importante mientras que el sabor y la temperatura son los aspectos más relevantes para todos los estratos, edades o género. Estos hallazgos se contradicen con los resultados de la cata a ciegas donde se encontró un pésimo reconocimiento de marca a partir del sabor; específicamente, con Póker, Águila, Águila Light y Andina hubo mucha confusión. Este resultado se asemeja a la experiencia del reto Pepsi (PepsiCo, 2005), en el que en degustaciones a ciegas se registra una preferencia por esta marca sobre Coca-Cola, aún cuando esta última se considera la líder del mercado. Al respecto, un estudio reciente donde se revisó la literatura sobre la paradoja del reto Pepsi, se indica que parece no haber una preferencia constante por el sabor de ninguna de las dos bebidas (Van y Miloyan, 2018). 
Figura 1. ¿Qué aspecto le parece más relevante al momento de escoger una cerveza?

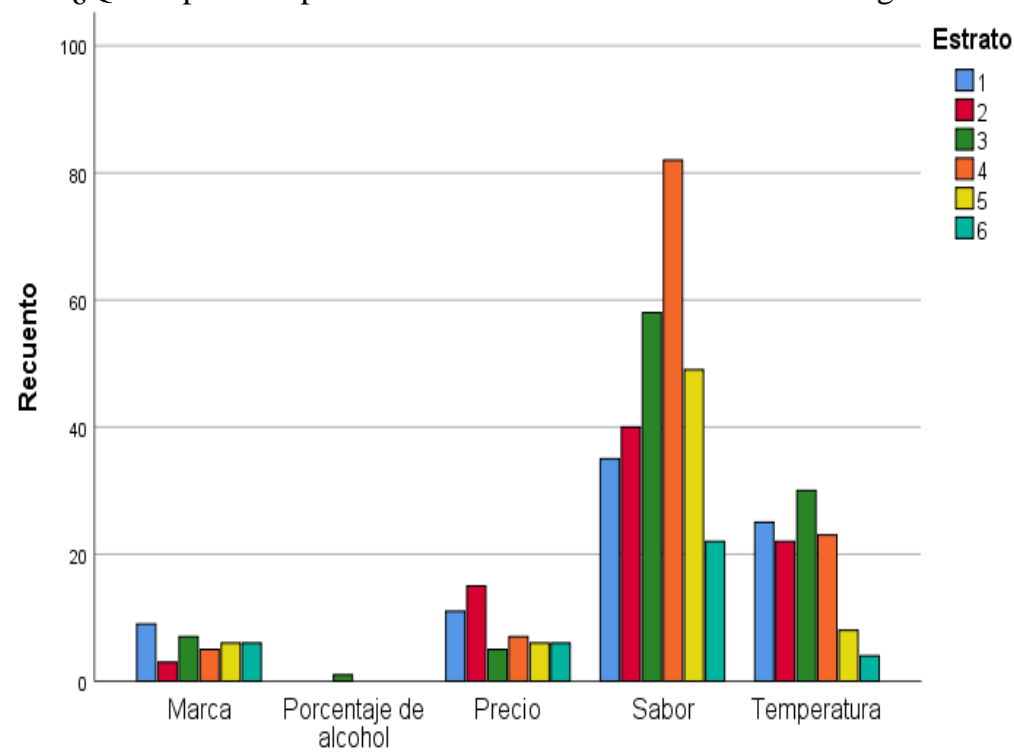

\section{CONCLUSIONES}

Los resultados de esta investigación sugieren que, aunque no es fácil reconocer el sabor característico de una marca de cerveza en una cata a ciegas, es posible establecer una asociación entre las marcas y el sabor que los consumidores prefieren. De acuerdo con esto, se podría argumentar que la marca (y no el sabor) es un factor primordial para el reconocimiento de la misma y su respectiva lealtad. Lo anterior permite pensar que el trabajo de branding influye de manera inconsciente en la toma de decisiones del consumidor, quien aún cuando no reporta la marca como un elemento importante, si se fía del valor de ésta para establecer sus preferencias, pues el sabor podría llegar a ser confundible. Aún así, es importante reconocer que las dimensiones sensoriales, como un gusto particular, ayudan a diferenciar y posicionar la marca en la mente del consumidor (Gobé, 2005; Hultén, 2011; Krishna, 2012).

En la industria cervecera, crear lealtad de marca es una tarea difícil dada la cantidad de oferentes que quieren obtener un porcentaje en la participación del mercado (Macias, 2016; America Retail, 2018). Según el reporte de Euromonitor (2020), los factores que podrían incidir en la selección de marca de cerveza en el contexto de la pandemia por COVID-19 incluyen un precio accesible y la disponibilidad del producto, pero se debería agregar una buena acción de comunicación de valor. Justamente, Póker ha sido la única marca que ha desarrollado una campaña publicitaria estimulando la responsabilidad social a través de conservar el distanciamiento personal ( 2 metros $=5$ petacas de cerveza), usando el hashtag "no es de amigos jugar a que no pasa nada". Así pues, no es de extrañar que Póker sea el líder indiscutible en el suroccidente colombiano pues es una marca que ha logrado crear plataformas emocionales (celebración del día de los amigos) que van más allá de los atributos funcionales del producto (sabor), conquistando con ello momentos de consumo que tienen que ver con la celebración de la amistad y la integración. 


\section{REFERENCIAS}

Aaker, D.A. (1996). Measuring brand equity across productsand markets, California Management Review, 38(3): 102-120.

Aaker, D. A. (2007). Construir marcas poderosas. Barcelona: Ediciones gestión 2000.

Aaker, D. A. (2015). Las marcas según Aaker, Orinda, California. URANO PUB Incorporated.

América retail (junio 2018). Colombia: Cinco tendencias de consumo de la cerveza artesanal. [En línea]. Recuperado de: https://bit.ly/2GgDBLp

Euromonitor. (2019). Las principales marcas de cerveza con mayor participación global. [En línea] Recuperado de https://bit.ly/2EGZIIA

Eurominitor. (2020). Beer in Colombia. Passport, Euromonitor International.

Gobé, Marc. (2005). Branding Emocional. Publicaciones Divine Egg.

González, E., Orozco, M. y Barrios, A. (2011). El valor de la marca desde la perspectiva del consumidor. Estudio empírico sobre preferencia, lealtad y experiencia de marca en procesos de alto y bajo involucramiento de compra. Contaduría y administración, (235), 217-239. Recuperado de: https://bit.ly/2P2tD31

Hulten, Bertil. (2011). Sensory marketing: The multi-sensory brand-experience concept. European Business Review. 23. 256-273.

Keller, K.L. (1993). Conceptualizing, Measuring and Managing Customer-Based Brand Equity. Journal of Marketing, vol.57, Nº1, 1-22. Recuperado de: https://bit.ly/1N10HO4

Kotler, P. y Armstrong, G. (2013). Fundamentos de Marketing. Trad. Roberto Luis Escalona García, México D.F: Pearson Education.

Krishna, Aradhna. (2012). An integrative review of sensory marketing: Engaging the senses to affect perception, judgment and behavior. Journal of Consumer Psychology 22(3).

La República (febrero 2019). La cerveza se lleva el 75\% del total de los gastos en licores que hacen los colombianos. [En línea] Recuperado de https://bit.ly/2Hj8gbM

La República (marzo 2020). Cerveza Andina fue uno de los ganadores de "Product of the Year 2020". [En línea] Recuperado de https://bit.ly/3ej2R2A

Masías, M, A. (2016). Estudio empírico de un shock de oferta transitorio en la lealtad a la marca en la industria cervecera en Chile. [en línea] Recuperado de https://bit.ly/2Lj7vTJ

Pepsico (2005). The Pepsi-Cola story. [En línea] Recuperado de: http://www.pepsi.com/PepsiLegacy_Book.pdf.

Roberts, K. (2004). “Lovemarks: The future beyond brands”. Powerhouse Books, New York. 
Roberts, K. (2006). "The Lovemarks effect: Winning in the consumer revolution”. Powerhouse Books, New York.

Schiffman, L. y Leslie, L. (2010). Comportamiento del consumidor. 10º edición, México D.F: Pearson Education.

Salomon. Michael R. (2008). La regla de los consumidores: ¿Qué es el comportamiento del consumidor? En Pearson Educación (Ed). Comportamiento del consumidor (Vol.7) (p.7). Recuperado de https://bit.ly/2lcGaSv

Tolba, A. H. y S. S. Hassan (2006). An integrative model for measuring customer-based brand equity and brand market performance. AMA Proceedings, 17, 114-115.

Van, D. G., \& Miloyan, B. (2018). The Pepsi Paradox: A review. Food Quality and Preference, 65, 194197. 